\title{
LIVES OF HIERARCHICAL TRIPLE SYSTEMS IN CLUSTERS AND IN THE FIELD
}

\author{
LUDMILA G. KISELEVA \\ Institute of Astronomy, \\ Madingley Road, Cambridge CB3 OHA, England
}

\begin{abstract}
Evolution with time of isolated hierarchical triple stars, stable from a dynamical point of view, is studied using analytical and numerical approaches. The results are applied to the study of the evolutionary cycle of hierarchical systems formed by dynamical capture in open clusters (up to 10,000 stars).
\end{abstract}

\section{Introduction}

Stars show a marked tendency to form systems of different multiplicity, starting from the smallest systems, binary and triple stars, up to globular clusters with $N \sim 10^{7}$. Different investigators have used different methods for the identification of multiple stars, and have arrived at somewhat different conclusions, but modern observations give a frequency of binary and multiple stars in the Galactic field of up to $70 \%$ (Gliese \& Jahreiss 1988, Batten, Fletcher \& McCarthy 1989, Duquennoy \& Mayor 1991), and between 5 and $15 \%$ of these systems are at least triple. Batten, Fletcher \& McCarthy (1989) claimed that about $20 \%$ of binaries in their sample can be at least triple. Among the 50 nearest stars $(\mathrm{G} / \mathrm{K} / \mathrm{M}$ dwarfs), from van de Kamp (1971) and Henry \& McCarthy (1990), are found 33 single, 13 binary and 4 triple stars. Duquennoy \& Mayor (1991) gives the following ratio of single : double : triple : quadruple systems among the 164 nearest G-dwarf stars: $1.5(91$ systems) $: 1(62): 0.105(7): 0.026(2)$. They also pointed out that the number of triple and quadruple systems may be larger.

A particularly large fraction of triple and quadruple systems can be observed among pre-main-sequence stars in star-forming regions. For example, Ghez et al. (1993) found that triples and quadruples comprise $14 \%$ 
of their sample for the Tau-Aur association. They estimate that the real frequency (taking into account the incomplete period coverage in their sample) may reach $\sim 35 \%$. Simon et al. (1995) identified at least 10 binaries, two triples and one quadruple among 35 young star targets in the Ophiuchus star-forming region, and 22 binaries and 4 triples among 47 systems in the Taurus region. Of course, some fraction of these systems may be unstable, and we observe them at the stage of the distant ejection of one companion.

The majority of observed triple and higher multiplicity systems are hierarchical, i.e. a close binary has a distant component, which may also be a binary. Another possibility is that a binary with a distant component has another even more distant component still. The majority of non-hierarchical triples (except a very few special cases like, for example, the Eulerian or Lagrangian configurations) are dynamically unstable, i.e. they eventually (usually, within several crossing times) disintegrate into a bounded binary and a detached single body which can escape to infinity: for a detailed discussion, see reviews by Anosova 1986, Heggie 1988, Anosova \& Orlov 1994.

Even for hierarchical triple-star systems, stability is not an easy question. There are a number of criteria to identify triple systems as stable or unstable, obtained analytically (e.g. Golubev 1967, 1968, Zare 1977, Szebehely \& Zare 1977, Marchal \& Bozis 1982) or numerically (e.g. Harrington 1975, 1977, Graziani \& Black 1981, Donnison \& Mikulskis 1992, 1995, Eggleton \& Kiseleva 1995a; hereinafter EK). These criteria differ from each other, sometimes rather significantly. Part of the reason for this is that 'stability' is a difficult concept to define and authors often use different stability definitions. In this paper we adopt the stability criterion of EK and also their definition of stability: that a hierarchical triple system is stable if it persists continuously for a very long time in the same hierarchical configuration (which excludes exchange as well as disintegration).

Known triple systems are not so numerous in open clusters as in the field, but the statistics are increasing due to the improvement of observational techniques, and to the systematic surveys undertaken at several observatories within the last few years. There is thus growing evidence for the existence of triple and even quadruple systems in open clusters, with a variety of characteristics. These systems are usually highly hierarchical. Triple (or even higher multiplicity) systems are found in the Pleiades (Mermilliod et al. 1992), the Hyades (Griffin \& Gunn 1981, Griffin et al. 1985, Mason et al. 1993), Praesepe (Mermilliod et al. 1994), M67 (Mathieu et al. 1990), and NGC 1502 (Mayer et al. 1994). The system in NGC 1502 contains an eclipsing massive binary SZ Cam $\left(m_{1}=13.7 M_{\odot}, m_{2}=9.7 M_{\odot}\right.$, $P=2.7 d$ ) which is the brightest member of the cluster. The variability of 
the orbital period of this binary has been known for some time, but only recently new high-dispersion spectra (Mayer et al. 1994) have allowed the third body to be identified. Because of its large mass (minimum $18.6 M_{\odot}$ ) and because of observed shifts in the third-body lines, this 'third body' can possibly be a binary, and the system as a whole may be a hierarchical quadruple system with $P_{\text {out }}=50.7 \mathrm{y}, e=0.77$.

Mermilliod et al. (1994) have summarised the data for 11 main-sequence triple systems known so far in open clusters, in which one component is a spectroscopic binary. Four of these systems contain a very close binary $\left(P_{\text {in }} \in(2.4,4.0) \mathrm{d}\right)$. Only 3 out of 11 outer orbital periods are known, and the least hierarchical system (vB 124 in the Hyades) has a period ratio $X=P_{\text {out }} / P_{\text {in }} \approx 250$.

Only one hierarchical triple system has been detected so far in globular clusters, but this is surely only the first step to the discovery of others which are likely to be present. This famous system in M4 contains the millisecond pulsar PSR B1620-26 (Backer et al. 1993, Thorsett et al. 1993; see also Rasio et al. 1995 and Hut 1995 for a discussion).

The above data indicate the importance of the numerical and analytical study of the formation and evolution of hierarchical systems in the Galactic field and in star clusters, which we discuss in the present paper. We particularly concentrate on hierarchical systems stable from the point of view of their internal dynamical evolution.

\section{Isolated stable hierarchical triple}

Dynamical stability requires that the ratio $X_{0}$ of outer period to inner period must be larger than a factor of $\simeq 3-6$, if both orbits are nearly circular and all three bodies are of comparable mass: Kiseleva, Eggleton \& Anosova 1994, Kiseleva, Eggleton \& Orlov 1994. More generally, for eccentric orbits, the outer periastron must be larger than inner apastron by a factor of $\simeq 2-16$ (EK; see also Harrington 1975). Table 1 gives, for a wide range of mass ratios, the minimum $Y_{0}$ necessary for stability in coplaner prograde orbits, where $Y_{0} \equiv R_{\mathrm{peri}}^{\text {out }} / R_{\mathrm{ap}}^{\mathrm{in}}$. This minimum $Y_{0}$ is not very sensitive to the two eccentricities, and so is not different by more than $\sim 20 \%$ from the value for two circular orbits, which is what is shown in the Table. In Table $1, \alpha=\log _{10} \frac{m_{1}}{m_{2}} \geq 0 S, \beta=\log _{10} \frac{m_{1}+m_{2}}{m_{3}}$, where $m_{1}, m_{2}$ are the masses of the components of the close binary and $m_{3}$ is the mass of the third body. The period ratio $X_{0}$ and the above distance ratio $Y_{0}$ are of course closely related by Kepler's law, given the two eccentricities and the two mass ratios.

Table 1 shows that for all systems with a massive distant component, i.e. $m_{3}>m_{1}+m_{2}, Y_{0}^{\min }>4$; and that for systems with $m_{1} \approx m_{2} \approx m_{3}$, 
TABLE 1. Minimum initial ratio $Y_{0}^{\text {min }}$ of semi-major axes $a_{\text {out }} / a_{\text {in }}$ for stability of triple systems with initially doubly-circular orbits.

$\begin{array}{rlllllllllll}\alpha & 0.0 & 0.2 & 0.4 & 0.6 & 0.8 & 1.0 & 1.2 & 1.4 & 1.6 & 1.8 & 2.0 \\ m_{1} / m_{12} & .50 & .39 & .28 & .20 & .14 & .09 & .06 & .04 & .025 & .016 & .01 \\ \beta\left(m_{3} / m_{12}\right) & & & & & & & & & & & \\ -2.0(100) & 15.53 & 15.62 & 15.85 & 15.90 & 16.00 & 16.05 & 16.14 & 16.15 & 16.14 & 16.17 & 16.20 \\ -1.8(63) & 13.35 & 13.49 & 13.65 & 13.77 & 13.82 & 13.87 & 13.91 & 13.94 & 13.98 & 13.98 & 14.00 \\ -1.6(40) & 11.48 & 11.64 & 11.74 & 11.88 & 11.99 & 12.04 & 12.03 & 12.10 & 12.11 & 12.11 & 12.10 \\ -1.4(25) & 9.87 & 10.01 & 10.18 & 10.29 & 10.34 & 10.42 & 10.44 & 10.44 & 10.46 & 10.48 & 10.51 \\ -1.2(16) & 8.57 & 8.65 & 8.81 & 8.86 & 8.95 & 8.99 & 9.00 & 9.05 & 9.07 & 9.07 & 9.08 \\ -1.0(10) & 7.34 & 7.42 & 7.55 & 7.66 & 7.72 & 7.76 & 7.82 & 7.83 & 7.86 & 7.86 & 7.87 \\ -0.8(6.3) & 6.31 & 6.41 & 6.51 & 6.61 & 6.67 & 6.72 & 6.76 & 6.79 & 6.79 & 6.79 & 6.81 \\ -0.6(4.0) & 5.39 & 5.51 & 5.61 & 5.70 & 5.76 & 5.82 & 5.85 & 5.87 & 5.89 & 5.90 & 5.91 \\ -0.4(2.5) & 4.61 & 4.72 & 4.82 & 4.91 & 4.97 & 5.02 & 5.06 & 5.08 & 5.09 & 5.10 & 5.10 \\ -0.2(1.6) & 3.95 & 4.03 & 4.11 & 4.20 & 4.27 & 4.31 & 4.34 & 4.36 & 4.37 & 4.38 & 4.38 \\ 0.0(1.0) & 3.37 & 3.46 & 3.47 & 3.60 & 3.63 & 3.67 & 3.70 & 3.72 & 3.73 & 3.74 & 3.74 \\ 0.2(0.63) & 3.11 & 3.12 & 3.10 & 3.22 & 3.04 & 3.05 & 3.09 & 3.12 & 3.14 & 3.15 & 3.16 \\ 0.4(.40) & 2.99 & 2.99 & 2.97 & 2.93 & 2.94 & 2.87 & 2.85 & 2.83 & 2.65 & 2.58 & 2.60 \\ 0.6(.25) & 2.88 & 2.87 & 2.87 & 2.84 & 2.81 & 2.80 & 2.73 & 2.72 & 2.71 & 2.70 & 2.69 \\ 0.8(.16) & 2.81 & 2.81 & 2.79 & 2.76 & 2.73 & 2.67 & 2.65 & 2.63 & 2.61 & 2.63 & 2.59 \\ 1.0(.10) & 2.74 & 2.74 & 2.72 & 2.70 & 2.36 & 2.33 & 2.29 & 2.26 & 2.22 & 2.23 & 2.21 \\ 1.2(.063) & 2.70 & 2.69 & 2.68 & 2.34 & 2.30 & 2.26 & 2.22 & 2.18 & 2.17 & 2.15 & 2.13 \\ 1.4(.040) & 2.67 & 2.35 & 2.33 & 2.29 & 2.25 & 2.20 & 2.18 & 2.14 & 2.07 & 2.05 & 2.04 \\ 1.6(.025) & 2.32 & 2.32 & 2.29 & 2.25 & 2.22 & 2.18 & 2.12 & 2.09 & 2.05 & 1.84 & 1.82 \\ 1.8(.016) & 2.31 & 2.31 & 2.27 & 2.22 & 2.19 & 2.15 & 2.10 & 2.08 & 2.03 & 1.83 & 1.63 \\ 2.0(.010) & 2.29 & 2.28 & 2.26 & 2.21 & 2.17 & 2.14 & 2.10 & 2.05 & 1.84 & 1.63 & 1.60\end{array}$

$3 \leq Y_{0}^{\min } \leq 4$. Thus a strongly hierarchical structure is required for dynamical stability in these cases. In this work we consider only triple systems with $Y_{0}$ significantly above $Y_{0}^{\mathrm{min}}$.

In dynamically stable hierarchical triples the distant component always pumps an eccentricity into the inner binary on a time scale shorter than the orbital period of the binary. In its turn, the binary also pumps an eccentricity into the outer orbit, although the inner orbit seems to be more sensitive to perturbations (Figs 1 and 2).

Fig. 1 shows that average values over time of the inner and outer eccentricities $\bar{e}_{\text {in }}$ and $\bar{e}_{\text {out }}$ increase rather smoothly as the period ratio $X_{0}$ decreases (but still does not approach too closely the critical value $X_{0}^{\min }$ for stability). However, such smooth behavior of $\bar{e}_{\text {out }}\left(X_{0}\right)$ and $\bar{e}_{\text {in }}\left(X_{0}\right)$ is not universal. Kiseleva, Eggleton \& Anosova (1994) found 'resonances' for some $(\alpha, \beta)$-pairs, i.e. $\bar{e}_{\text {out }}$ or $\bar{e}_{\text {in }}$ can rapidly increase and then decrease again in a narrow range of $X_{0}$. For yet other $(\alpha, \beta)$-pairs the resonance is 'disrup- 

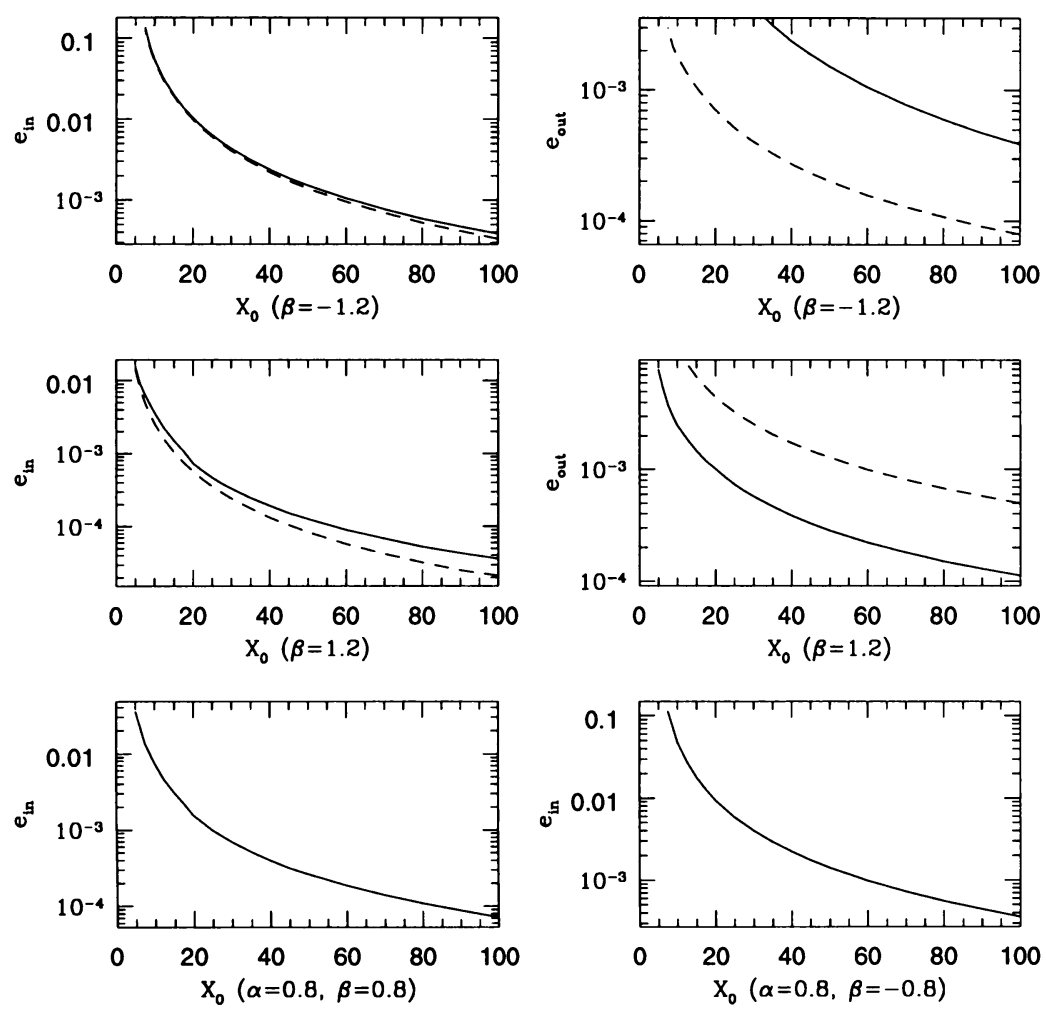

Figure 1. The mean values over time of the inner and outer eccentricities in hierarchical triples with coplanar initially circular orbits, as functions of the initial period ratio $X_{0}$. In upper and middle panels dashed lines show results for equal mass inner binaries (i.e. $\alpha=0$ ) and solid lines for binaries with a rather significant mass ratio $\frac{m_{1}}{m_{2}} \approx 16$ (i.e. $\alpha=1.2$ ).

tive': for some rather narrow range of $X_{0}$ the system breaks up, typically by ejection of a distant component, even though the system appears to be stable over very long time intervals at smaller as well as larger values of $X_{0}$; although of course the system will eventually break up at some smaller $X_{0}$ still. Fig. 1 shows that for $X_{0} \geq 20$ the initial orbital elements are preserved in both binaries to better (usually much better) than $1 \%$, so that inner and outer subsystems can be considered as rather unaffected by each other. However, Heggie (1996) has shown analytically that for non-coplanar triple systems there is a secular periodic change of the inner eccentricity which must over a sufficiently long time (which depends on both inner and outer periods, initial eccentricities, and masses) reach its maximum value $e_{\mathrm{in}}^{\max }$. This value does not depend on any parameters of the triple system 

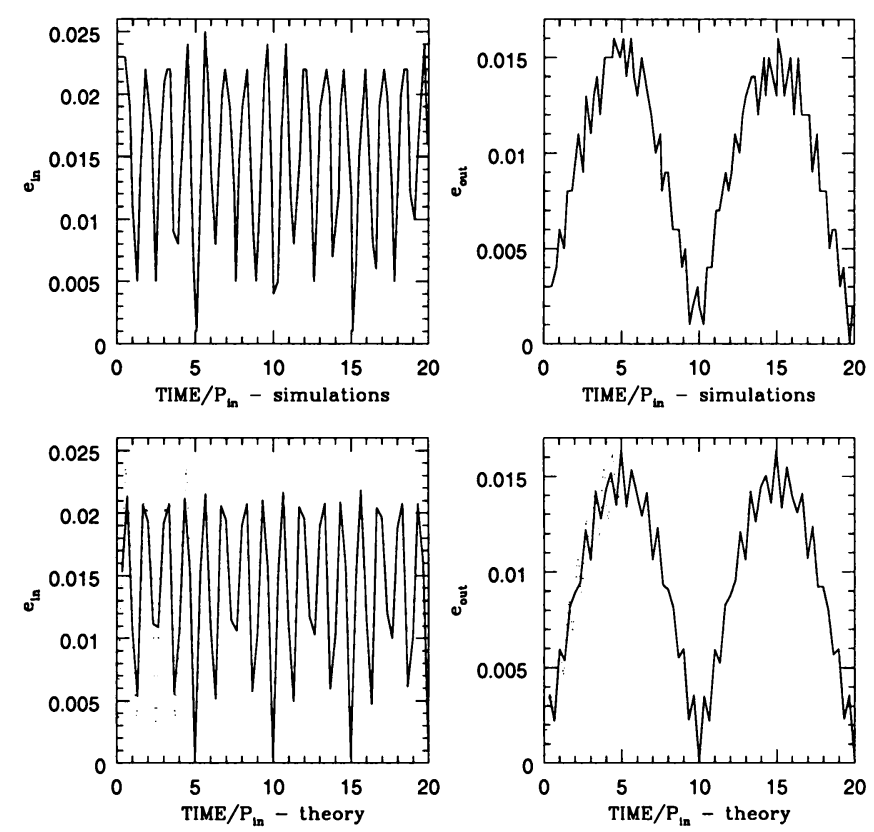

Figure 2. The fluctuations of inner and outer eccentricity with time for a system with $m_{1}=m_{2}=m_{3}$ and $x_{0}=10$. The upper panel shows results of numerical 3-body simulations and the lower panel gives data produced using Heggie's analytical approach (see text).

except the relative inclination $i$ between two orbital planes (other parameters affect only the time scale) If $i \approx 90^{\circ} e_{\mathrm{in}}^{\max } \approx 1$ and the two stars may collide or at least have a very strong tidal interaction. This affect cannot be neglected in the numerical study of triple stars in clusters which we will discuss in the next Section. Fig. 1 also show that the changes of the orbital characteristics of the inner binary in practice do not depend very much on the mass ratio $\alpha$ of the components of this binary. However, $e_{\text {out }}$ depends on $\alpha$ rather strongly.

Fig. 2 presents the behaviour in time of instantaneous $e_{\text {in }}$ and $e_{\text {out }}$ for a stable $\left(X_{0}=10\right)$ triple with three components of equal masses for numerical simulations (upper panel) and the analytical approximation by Heggie (private communication) based on 1st order perturbation theory. Fig. 2 shows a resonably good aggreement between these two approaches, especially for $e_{\text {out }}$. Although the fine details of both functions differ, the amplitudes $e_{\text {out }}^{\max }$ and periods of fluctuations are nearly the same. For $e_{\text {in }}$ the difference between the two approaches is more significant. The BulirschStoer integration procedure used in numerical simulations does not allow us to have more than 4-5 outputs per inner orbit, and so for uniformity we 
used the same sampling for the analytical curves; however the small dots on the lower panel correspond to 20 outputs per inner orbit.

\section{Hierarchical systems in open clusters}

A few numerical simulations for clusters of $500-10,000$ stars with different fractions of primordial binaries were performed, using the $\mathrm{N}$-body code NBODY4 (Aarseth 1996) on HARP in IoA. The procedure for identification and observation of dynamically formed hierarchical systems in clusters is described in Kiseleva et al. (1995): a newly formed hierarchical system (triple or quadruple in the sense that the distant component can itself be a binary) is recorded if it satisfies the EK stability criterion and at the same time the distant star forms a hard binary with respect to the centre of mass of the inner binary. Usually there are more triples than quadruples; however in some runs the fraction of quadruples may be up to $45 \%$. Hierarchies can be destroyed because of perturbing effects of the remaining cluster stars or/and some stellar evolutionary effects.

Possible destructive effects of the secular increase of the inner eccentricity in non-coplanar triple systems, as described above, have not been taken into account so far and the inner orbit was 'frozen' as long as the hierarchy existed. However, numerical simulations show (Fig. 3) that relative inclinations $i$ of the two orbital planes of dynamically formed hierarchical systems in clusters are uniformly distributed between $0^{\circ}$ and $180^{\circ}$ (keeping in mind that $n(i) d i \propto \sin i d i)$, and therefore the actual fraction of systems with $i \approx 90^{\circ}$ is rather significant. The factor which can significantly reduce the influence of 'Heggie's effect' on the inner binary is the time scale of this effect. Some statistical properties of hierarchical systems in clusters are shown in Fig. 4. Such factors as a large ratio of semiajor axes, massive inner binary $\left(m_{1}+m_{2} \geq 2.5 M_{\odot}\right)$, and relatively low-mass distant component (on average $\left.m_{3} \sim(0.2-0.6) m_{\text {in }}\right)$ should increase the time interval which is required in order to reach $e_{\mathrm{in}}^{\max }$. In this case the life-time of a hierarchy, which is typically between 1 and 3 Myrs although it can reach 10 Myrs in some cases, may not be long enough. Also the tidal circulization which time scale can be comparable or even less that one of 'Heggie's effect' can play an important role and close binary, probably, cannot even approach their $e_{\mathrm{in}}^{\max }$. This study has just begun and we cannot yet draw any final conclusion as to how the fate of the close binary may be affected over a long time by the presence of the very distant companion. Fig. 5 presents data from the last part of the run for the model cluster with 10,000 stars and 500 primordial binaries. The left-hand panel shows the distibution of $e_{\mathrm{in}}^{\max }$, with $e_{\mathrm{in}}^{\max }$ calculated at the time when the hierarchy was formed. More than $15 \%$ of systems may reach $e_{\mathrm{in}}^{\max } \in(0.9 ; 1)$ if they are given enough time. 


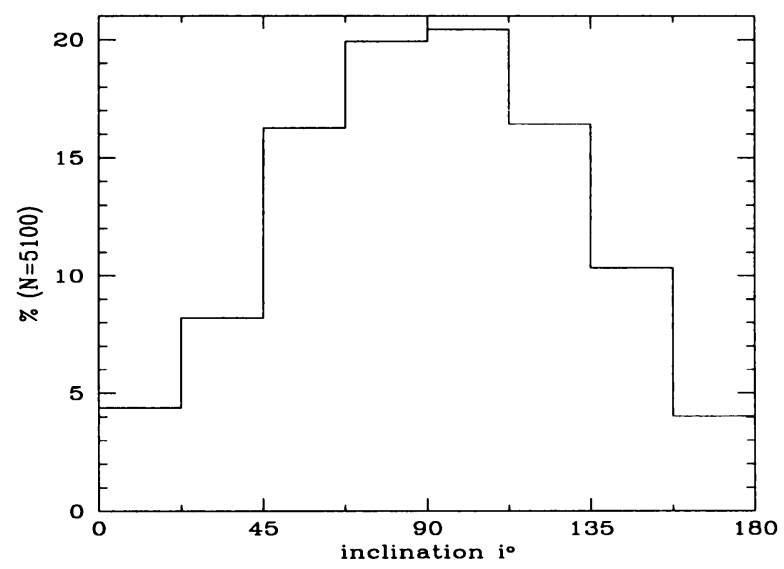

Figure 3. Distribution of relative orbital inclinations $i$ for hierarchical triple and quadruple systems in two runs for clusters of $N=5100$ stars with 100 primordial binaries
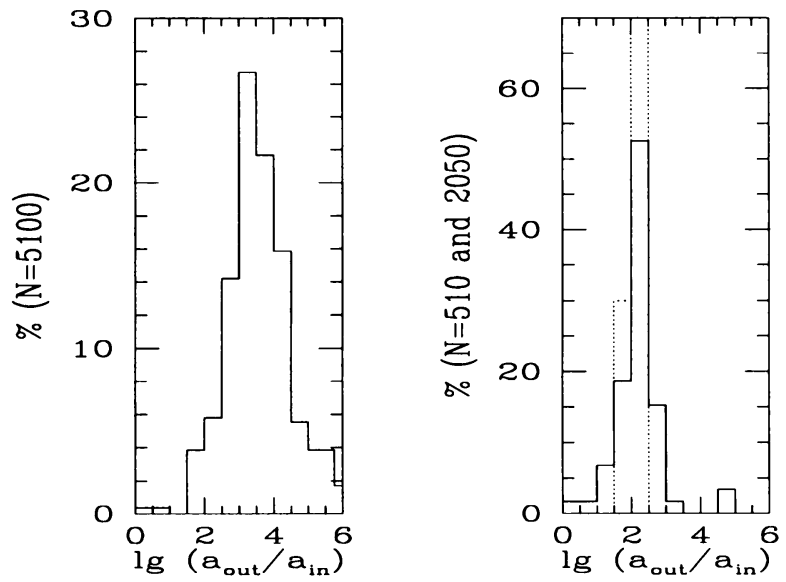

Figure 4. Distribution of the ratio outer/inner semi-major axis $\left(\log a_{\text {out }} / a_{\text {in }}\right)$ for models with 5100 stars (left-hand panel); 510 stars with 10 primordial binaries (dots) and 2050 stars with 50 primordial binaries (solid line) on the right-hand panel

Note that at the moment of hierarchy formation all inner binaries had circular orbits (most likely due to tidal circularization), although about $25 \%$ of triples and $39 \%$ of quadruples contained a non-primordial inner binary. The right-hand panel shows that if systems are allowed to reach their $e_{\mathrm{in}}^{\max }$, a few percent of inner binaries would suffer collisions or very strong tidal effects at the periastrons of their orbits.

So far, we have not followed the processes of formation of hierarchical systems in clusters in detail. The most probable mechanism is the presence 

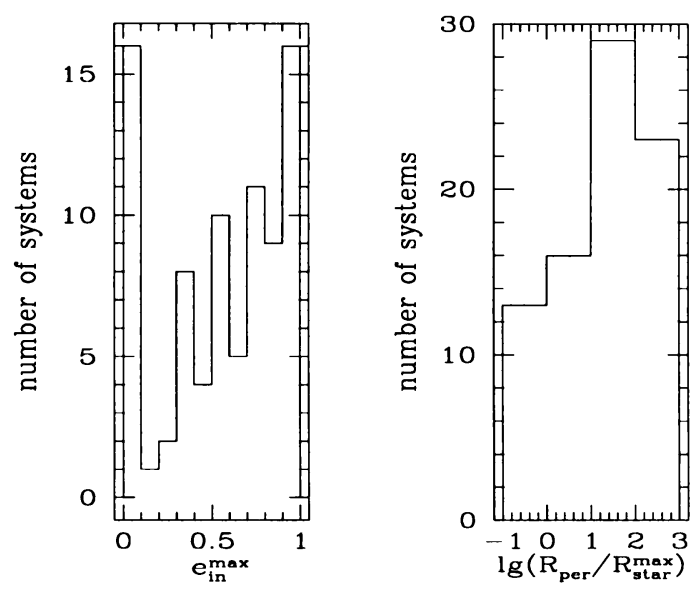

Figure 5. Distributions of maximum values of inner eccentricity due to the secular effect of the distant component (left), and of minimum (periastron) separations between stars in the outer binary in units of their maximum stellar radius

of primordial or, at later evolutionary stages, exchanged binaries (which can build up to $50 \%$ of all close binaries in hierarchies in some numerical runs), along with binary-binary interaction. However, our distribution of the ratio $a_{\text {out }} / a_{\text {in }}$ (Fig. 4), especially for big clusters with $N>5000$, differs very much from the results of binary-binary scattering experiment (McMillan, Hut \& Makino 1991). It appears from numerical experiments that in large clusters at a late stage of their evolution new hierarchical systems can be formed via repetitive triple-binary and triple-triple exchanges. Outer eccentricities - most systems have very eccentric outer orbits with $\left.e_{\text {out }} \in(0.7 ; 0.98)\right)$ and the periods for hierarchical systems produced in our models, are in reasonable agreement with the observations referred to above of multiples in open clusters, within the limited statistics for the latter. Let us note finally that at least one hierarchical system (in large clusters there can be up to 5 hierarchies at the same time) is present in a open cluster during about $20 \%$ of its life history and at all evolutionnary stages after the core collapse.

\section{Acknowledgements}

My special thanks go to S. Aarseth without whom the last section of this article would not exist. I am also very grateful to D. Heggie for allowing me to use his unpublished formulae, some of which were derived specially to answer my questions. Many thanks also to P. Eggleton and R. de la Fuente Marcos for fruitful discussions and cooperation, to NATO, the Isaac Newton 
Trust, and the IAU for their financial support and to IoA in Cambridge for its hospitality.

\section{References}

Aarseth S. J. (1996) in E. F. Milone, ed., Binaries in Clusters, ASP series, in press

Anosova, J.P. (1986) Astrophys. Space Sci. 124, 217

Anosova, J. P. and Orlov, V. V. (1994) Celest. Mech. 59, 327

Backer, D. C., Foster, R. S., and Sallmen, S. (1993) Nature 365, 81

Batten, A. H., Fletcher, J. M., and MacCarthy, D. G. (1989) Publ. Dom. Astrophys. Obs. 17,1

Donnison, J.R., Mikulskis, D.F. (1992) Mon.Not.R.Astr.Soc. 254, 21

Donnison, J.R., Mikulskis, D.F. (1995) Mon.Not.R.Astr.Soc. 272, 1

Duqennoy, A. and Mayor, A. (1991) Astron. Astrophys. 248, 485

Eggleton, P. P. and Kiseleva, L. G. (1995) Astrophys. J., in press

Ghez, A. M., Neugebauer, G., and Matthews, K.: 1993, Astron. J. 106, 2066

Gliese, W., Jahreiss, H. (1988) Astrophys. Space Sci., 142, 49

Golubev, V. G. (1967) Dok. Akad. Nauk USSR, 12, 529

Golubev, V. G. (1968) Dok. Akad. Nauk USSR, 13, 373

Graziani, F. and Black, D. C. Astrophys. J., 251, 337

Griffin, R. F. and Gunn, J. E. (1981) Observatory 106, 35

Griffin, R. F., Gunn, J. E., Zimmerman, B. A., and Griffin, R. E. M. (1985) Astron. J. 90, 609

Harrington, R.S. (1972) Celest. Mech., 6, 322

Harrington, R.S. (1975) Astron. J., 80, 1081

Heggie, D.C. (1988) in M. Valtonen, ed., The Few-Body Problem, Reidel, Dordrecht, p. 213

Heggie, D.C. (1996), this Proceedings

Henry, T. J. and McCarthy, D. W. (1990) Astrophys. J. 350, 334

Hut, P. (1995) in E. P. J. van den Heuvel, ed., Compact Stars in Binaries, in press

Kiseleva, L. G., Aarseth, S. J., Eggleton, P. P., and de la Fuente Marcos, R. (1996) in E. F. Milone, ed., Binaries in Clusters, ASP series, in press

Kiseleva, L. G., Eggleton, P. P., and Anosova, J. P. (1994) Mon. Not. R. Astron. Soc. 267, 161

Kiseleva, L. G., Eggleton, P. P., and Orlov, V. V. (1994) Mon. Not. R. Astron. Soc. 270, 936

Mason, B. D., McAlister, H. A., Hartkopf, W. I., and Bagnuolo, W. G., Jr. (1993) Astron. J. 105, 220

Mathieu, R. D., Latham, D. W., and Griffin, R. F. (1990) Astron. J. 100, 1859

Mayer, P., Lorenz, R., Chochol, D., and Irsmambetova, T. R. (1994) Astron. Astrophys. 288, L13

McMillan, S., Hut, P., and Makino, J. (1991) Astrophys. J., 372, 111

Mermilliod, J. C., Rosvick, J., Duquennoy, A., and Mayor, M. (1992) Astron. Astrophys. 265, 513

Mermilliod, J. C., Duquennoy, A., and Mayor, M. (1994) Astron. Astrophys. 283, 515

Rasio, F. A., McMillan, S., and Hut, P. (1995) Astrophys. J. Letters 498, L33

Simon, M., Ghezz, A. M., Leinert Ch. et al. (1995) Astrophys. J. 443, 625

Szebehely, V. and Zare, K. (1977) Astron. Astrophys., 58, 145

Thorsett, S. E., Arzoumanian, Z. and Taylor, J. H. (1993) Astrophys. J. 412, L33

van de Kamp, P. (1971) Annu. Rev. Astron. Astrophys. 9, 103

Zare, K. (1976) Celest. Mech., 14, 73 